\title{
Responsabilidad \\ Social Empresarial
}

El asunto de la relación trabajo - familia
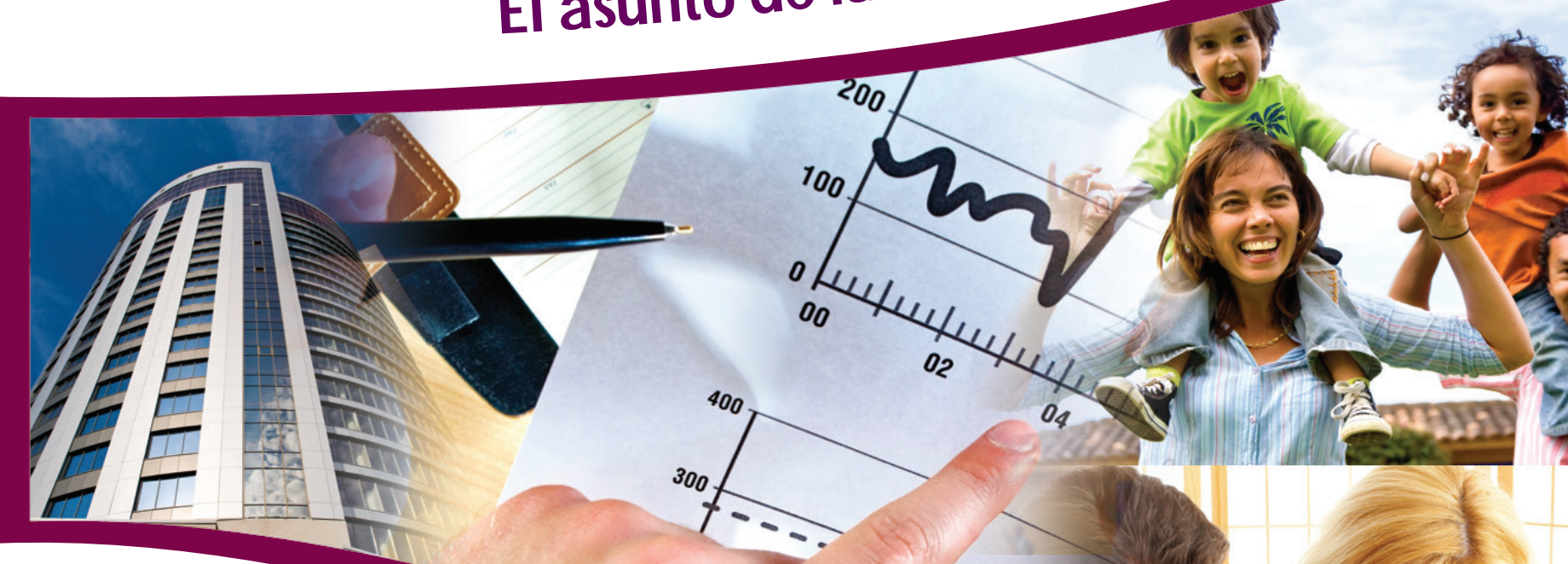

Francisco Pacheco Sempértegui

Resumen-El artículo introduce el tema de la Responsabilidad Social Empresarial, su concepto, historia y desarrollo en el Ecuador. Menciona las normas relacionadas con el tema y se enfoca específicamente al tema de la relación trabajo familia en las empresas. Introduce el concepto de Empresas Familiarmente Responsables.

Palabras clave- Empresas familiarmente responsables, historia de la RSE, normas de la RSE, responsabilidad social empresarial - RSE, recursos humanos, relación trabajo - familia.

En estos días se escucha frecuentemente hablar de la Responsabilidad Social Empresarial - RSE. En nuestro país, este es un tema que aún está emergiendo y que empieza a introducir algunos aspectos que hasta ahora las empresas no habían tomado en cuenta, por ejemplo la relación trabajo familia de los empleados y administradores. Este artículo recoge los conceptos fundamentales de la RSE, algo de su historia y discute el asunto de la familia y su relación con el trabajo.
Primero definamos qué es la

RSE. En muchas ocasiones, éste término se confunde con filantropía o con algunas actitudes generosas de las empresas. Su concepto no ha logrado un consenso entre las organizaciones. Por ejemplo la World Business Council for Sustainable Development (Consejo Empresarial Mundial para el Desarrollo Sostenible) define a la Responsabilidad Social Empresarial como: "El compromiso de la empresa para contribuir al desarrollo económico sostenible, trabajar con los empleados, sus familias y la comunidad local en general para mejorar la calidad de vida" (Moller, citado en Saguma, 2007, p16). En cambio el Libro Verde de la Unión Europea (2007) precisa que la RSE es la integración voluntaria

1 Francisco Pacheco Sempértegui, Docente de Posgrados de la 
por parte de las empresas de las preocupaciones sociales y medioambientales en sus operaciones comerciales y sus relaciones con sus interlocutores. Otra definición interesante, que toma en cuenta el tema que aquí tratamos, es la desarrollada por la Fundación Prohumana de Chile (2008). Esta dice que la RSE es la contribución al desarrollo humano sostenible a través del compromiso y confianza con sus empleados y familia, la sociedad en general y la comunidad local en pos de mejorar su capital social y calidad de vida. Finalmente podemos decir que la RSE es un concepto que implica la "obligación"2 de las organizaciones, que desarrollan algún tipo de actividad económica, de mantener una conducta respetuosa de las leyes, la ética y el medio ambiente. Además, de contribuir al desarrollo sostenible; lo cual implica la adopción de una nueva ética en su actuación en relación a la naturaleza, la sociedad en general y sobre todo con la vida de sus grupos de interés ${ }^{3}$ y sus familias.

\section{Responsabilidad Social Empresarial: breve historia}

Resumamos algo de su historia. La idea de "la responsabilidad social empresarial" comenzó a ser asumida por numerosos actores del contexto político y económico mundial, incluidos instituciones financieras, empresas, organizaciones, agencias de desarrollo, facultades de administración, inversionistas y algunos gobiernos, a finales de la década de los noventa. Aunque este concepto es relativamente novedoso sus raíces se identifican en diversas concepciones acerca de la gestión empresarial en los Estados Unidos entre los años 1950 y 1960, donde la responsabilidad social era considerada una obligación moral y responsabilidad personal del empresario (revista Fututos, 2008). Ya en 1953, Howard Bowen hace mención de este tema a través de la publicación de Responsabilidades Sociales del Hombre de Negocios en donde menciona que las empresas deberían tomar en cuenta las consecuencias sociales de sus decisiones. En la década de los años 60, la RSE se mantenía aún como un concepto que se discutía en círculos intelectuales, mientras que en los años setentas y ochentas se insertó en la gestión de negocios de manera voluntaria (Chacón, 2006, p.90), aunque aún cuestionada por algunos economistas influyentes como Milton Friedman, ganador del premio Nobel de Economía, quien algún momento llegó a decir "¿Cómo definirlo que es socialmente responsable? ¿Por qué tiene una corporación que decidir lo que es? Ese no es su campo, no es lo que le pidieron los accionistas, creo que se están saliendo de su área y ciertamente no es democrático" (Mark A. \& Jennifer A.).

En América Latina, la primera organización que promovió la RSE fue la Asociación de Reguladores Cristianos de las Compañías del Brasil (ADCE), creada en 1965. En los años 90 se crearon una serie de instituciones encargadas de promover la RSE y en el Ecuador nació el Consorcio Ecuatoriano de RSE - CERES en el 2002. La presencia de distintas organizaciones nacionales ayudó a que el movimiento de la RSE creciera en América Latina y en nuestro país (Chacón S., p.102).

\section{La necesidad de la RSE}

Si nos preguntamos por qué es necesaria la RSE, debemos acudir a algunas cifras preocupantes de inequidad global. Por ejemplo, si todos los habitantes del mundo formaran una aldea de tan solo 100 personas, entonces:

- 80 personas vivirían en viviendas precarias.

- 66 no tendrían agua potable para beber.

- 66 nunca habrían realizado una llamada telefónica.

- 50 sufrirían de desnutrición.

- 6 poseerían la mitad de la riqueza.

- 1 poseería educación universitaria.

- 1 tendría su propio computador ${ }^{4}$

Ante estas cifras, los creadores de riqueza (las empresas y los empresarios) empiezan a cuestionarse cuál debe ser su nuevo papel en la sociedad y de esta reflexión nacen las iniciales preocupaciones de la Responsabilidad Social Empresarial.

\section{Normas de Responsabilidad Social Empresarial}

En el tema de la RSE aún no existe una norma que sirva de referencia. Existe la norma OHSAS 18000, que trata los temas de salud y seguridad en el trabajo. También está la norma SA 8000 (Social Accountability) que se basa en la Convención de los Derechos del Niño y la Convención sobre la Eliminación de toda forma de Discriminación contra la Mujer de las Naciones Unidas, las recomendaciones de la Organización Internacional del Trabajo y la Declaración Universal de los Derechos Humanos. Es de carácter voluntario y puede ser aplicada en cualquier país y empresa. Está basada en las normas ISO 9000 e ISO 14000 y requiere de una auditoría externa a fin de comprobar su aplicación (Saguma M, p.24). Adicionalmente la Organización Internacional de Estandarización (ISO) está elaborando la norma ISO 26000, que sería una guía para la responsabilidad social. Aún está en discusión y se espera esté lista en el año 2009. Igualmente se considera que esta norma será de carácter voluntario.

\section{El asunto de la relación trabajo - familia y la RSE}

Enfocándonos ya en el tema específico de este artículo, la relación empresa - familia, nos preguntamos ¿desde cuándo las empresas se preocupan por el tema de familia? Mi opinión personal es que en algunos (¿muchos?) casos, no es simple altruismo sino, por supuesto, tiene relación con el concepto de eficiencia económica y la búsqueda de lucro, que es uno de los objetivos más importantes a la hora de constituir una empresa. Las organizaciones se han dado cuenta que un empleado que no tiene una buena relación familiar (con su 
cónyuge, hijos padre, madre o algún otro miembro del círculo familiar) no rinde lo máximo en su trabajo, es decir no es lo eficiente que podría (o debería) ser. Y esto es lógico porque el ser humano no está dividido en parcelas, independientes unas de otras, sino que es un solo ser integral. Cuando va a trabajar, muchas veces no puede dejar sus problemas en casa (aunque sea muy "profesional"), como tampoco puede dejar los problemas de la empresa en su trabajo, cuando llega a casa. Por lo dicho anteriormente, vaticino entonces que la preocupación de la empresa por los asuntos de la familia irá en aumento porque está alineada con sus principales objetivos económicos, la rentabilidad, el crecimiento y la supervivencia.

Entre otros, existen dos temas involucrados en el asunto de la relación trabajo. El primero es la necesidad de que más miembros de la familia se involucren en el mundo del trabajo remunerado (padre y madre, por ejemplo) y la segunda la duración de las jornadas de trabajo. El primero no es un asunto que involucre directamente a la Responsabilidad Social Empresarial. Respecto al segundo, es muy conocida la queja de los empleados y trabajadores que, en las actuales circunstancias de dificultades económicas para las familias, por un lado, y en las de una competencia más intensa, por el lado de las empresas, los horarios del trabajo son más extensos de lo que originalmente constan en los contratos. Chiriboga (2007) al respecto menciona "en lo que compete a la parte administrativa el horario es de 7:45 a 17:00 el mismo, que nunca se cumple en el 99\% del personal por sobrecarga operativa", además que "por otra parte la realidad en lo que respecta a las horas extras ... en la mayoría de los casos el promedio es de más de 12 horas por trabajador por semana" (pp. 147- 148). Afortunadamente, este asunto ya se toma en cuenta actualmente en una norma, por ejemplo, en la mencionada SA 8000 (Sección 7.1) se dice que:

La semana de trabajo normal será como la defina la ley, pero no excederá las 48 horas. Se otorgará al personal por lo menos un día libre en cada período de siete (7) días laborados. Todo trabajo que implique horas extras será reembolsado con paga adicional superior a la de las horas normales, pero bajo ninguna circunstancia deberá exceder 12 horas por empleado por semana.

Este es un asunto que las empresas que implementen la RSE deberán tomar en cuenta, entre otros temas introducidos por la norma mencionada.

\section{Empresas Familiarmente Responsables}

El IESE (2001), una de las escuelas de negocios más prestigiosas de Europa con sede en Barcelona España ${ }^{5}$ desarrolló el concepto de "empresas familiarmente

5 En España, el 5 de noviembre de 1999 se expidió una ley para la conciliación de la vida familiar y laboral de las personas trabajadoras. Sin embargo los españoles se consideran "atrasados" respecto a Europa en el tratamiento de estos temas. responsables". En su propuesta, define una empresa familiarmente responsable como la que "se esfuerza para que sus empleados puedan realmente mantener un equilibrio sano entre su trabajo y su familia". Según el IESE, este equilibrio "es esencial para el bienestar de sus empleados y su capacidad de compromiso con la empresa". "Las empresas son cada vez más conscientes de que el equilibrio personal repercute en el rendimiento y la productividad", dice Nuria Chinchilla, profesora del IESE, una de las abanderadas de este nuevo concepto gerencial. El concepto de empresas familiarmente responsables está creciendo, aunque se trate de algo todavía poco conocido, sobre todo en el Ecuador.

Las políticas que podrían implementar las empresas familiarmente responsables son, entonces: una jornada laboral flexible, en donde los empleados trabajan ocho horas, pero deciden cuando empiezan y cuando acaban; la reducción de jornada, con la posibilidad de trabajar menos horas a la semana, con un menor salario; una jornada compartida, por la cual dos trabajadores a tiempo parcial pueden completar una jornada completa; o una jornada comprimida, si se dedica más horas durante la semana con el fin de obtener una tarde libre.

Un segundo grupo de políticas se refieren a los permisos: tiempo adicional por maternidad o paternidad, cuando por necesidad del padre o la madre, éstos permanecen más tiempo en casa de lo que legalmente tienen derecho; ${ }^{6}$; permiso para cuidados especiales de niños o ancianos enfermos. En ambos casos, sin derecho a cobrar por ese período. También, las empresas podrían conceder a sus empleados un tiempo sabático (tres meses a un año), durante el cual se le pagaría un porcentaje de su salario.

Finalmente, en cuanto a políticas existe un tercer grupo que pretende dar al empleado la posibilidad de trabajar desde casa, lo que requiere la tecnología necesaria para conectarse con la empresa; o a utilizar videoconferencias para encontrarse con clientes o colaboradores y así reducir la necesidad de viajes de negocios.

\section{Y en el Ecuador, ¿qué pasa?}

Es reconfortante saber que las empresas empiezan a tomar en cuenta éste y otros temas relacionados con la familia en distintos países, por ejemplo en el Ecuador ya existen empresas, que se preocupan por las familias de sus empleados, es el caso de Interquimec, empresa que fabrica resinas, adhesivos y formol. La empresa se involucró en el tema desde hace seis años y promueve una nueva cultura

6 En el Ecuador (Febrero del 2009) se aprobó la Licencia por Paternidad en donde por primera vez se permite a los padres obtener unos días de permiso (de 10 a 25) cuando nacen sus hijos, lo que constituye un avance en el tema, aunque varias voces han expresado sus dudas sobre el tema. También se aprobó una licencia de 25 días para el padre, para atender los casos de hijos hospitalizados o con patologías. 
empresarial que tiene como eje la atención a los trabajadores. La empresa ha patrocinado charlas de orientación familiar, una escuela para padres y terapias familiares. Actualmente está en proceso de implementar un Diplomado en Familia dirigido a los empleados (Líderes, 7 de abril del 2008).

También se puede mencionar que la propuesta de Empresa Familiarmente Responsable se aplica en la Compañía de Seguros Ecuatoriano Suiza (El Universo, 23 de febrero del 2009), en donde el concepto implica, entre otros asuntos "Tener políticas sensibles, sí, pero el compromiso de la persona con la organización hace que se reduzca la rotación de personal. La gente empieza a valorar a la empresa no solo por salario o los puestos. Al ser tu familia tu principal responsabilidad eso no tiene precio".

Finalmente creemos que este tema, el de la familia y su relación con el mundo del trabajo remunerado, seguirá creciendo en el futuro y vaticinamos que, así mismo como ha sucedido con la familia, ocurrirá con la espiritualidad, otro aspecto importante que ha sido muchas veces despreciado, pero que será importante en el presente siglo, como lo han pronosticado los expertos en prospección, pero este ya es asunto de un nuevo artículo.

\section{Bibliografía}

- Battaini Gabela, M.N. (2008). Modelos de responsabilidad social corporativa (RSC). Quito: Pontificia Universidad Católica del Ecuador, Escuela de Trabajo Social.

- Chacón S. (2006). La Responsabilidad Social Corporativa como un instrumento de desarrollo humano en el Ecuador y de sostenibilidad Empresarial, caso: empresas del consorcio CERES, Tesis de Licenciada, Facultad de Comunicación, Lingüística y Literatura, PUCE.

- Chiriboga M. (2007). Calidad de Vida Laboral en las Empresas General Motors del Ecuador y Omnibus BB Transportes S.A.: Intervención de Gestión Social acorde a la norma SA 8000 de Responsabilidad Social, Tesis Magíster, Escuela de Trabajo Social, PUCE.

- El Universo, extraído el 23 de febrero del 2009 desde http://www.eluniverso.com/2008/08/24/0217/865/ COBAB6A170754F459E179159C9DCBA6F.html.

- Fundación Prohumana (2008). Acceso el 4 de agosto del 2008, 16h45 desde http://www.prohumana.cl/index. php?option=com_content\#\&task=view\&id=2479\&ltemid $=176$.

- Libro Verde de la Unión Europea, extraído el 2 de agosto del 2008 desde http://ec.europa.eu/employment_social/soc-dial/ csr/greenpaper_es.pdf.

- Mark A. \& Jennifer A. (Productor ejecutivo), La Corporación [Programa televisivo].

- Navarro García, F. (2008). Responsabilidad Social Corporativa. Madrid: Editorial Esic.

- Perdiguero, T. G. (2003). La responsabilidad social de las empresas en un mundo global. Barcelona: Editorial Anagrama.
- Revista Futuros, extraído el 1 de agosto de 2008, desde http:// www.revistafuturos.info/futuros17/resp_soc_emp2.htm.

- Revista Líderes (2008, abril 7). El empleado y su familia forman el eje, 543, p.13.

- Saguma, D (2007). La Responsabilidad Social Empresarial como mecanismo generador de bienestar humano y prestigio corporativo de. Tesis de Licenciada, Facultad de Comunicación, Lingüística y Literatura, PUCE.

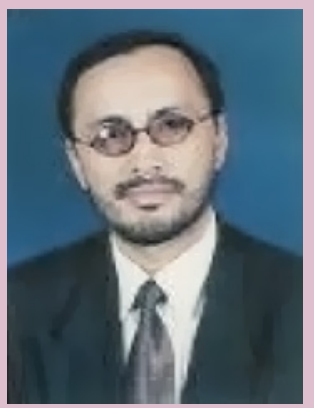

Autor. Francisco Pacheco Sempértegui,

Es actualmente Docente de la cátedra "Ética en los Negocios" de la Maestría en Auditoría y Finanzas de la Universidad Tecnológica Equinoccial - UTE. Director de la organización Homini, entidad dedicada al entrenamiento en Familia, Habilidades Gerenciales, Trabajo en Equipo, Liderazgo y Valores, así como profesor de Desarrollo Organizacional y Dirección Estratégica de la Pontificia Universidad Católica del Ecuador, en la Facultad de Comunicación y la Escuela de Gestión Social. Ha sido consultor en familia, liderazgo, valores, e inteligencia emocional para Petroecuador, U.E. Julio Pierregrosse, Helmerich \& Payne, Grupo el Comercio, entre otras empresas, así como una veintena de instituciones educativas.

Se ha desempeñado como Profesor de Liderazgo, Cultura de Valores, Toma de Decisiones, Estrategia y del área de Finanzas en la Universidad Internacional del Ecuador, Escuela Politécnica Nacional, Escuela Politécnica del Ejército, Universidad Internacional SEK y la Universidad del Pacífico. Fue Decano de la Facultad de Ciencias Administrativas y Económicas de la Universidad Internacional del Ecuador. Recibió entrenamiento en la metodología de casos reales (enseñanza y escritura) en el Instituto Centroamericano de Administración de Empresas - INCAE, la Escuela de Negocios Ivey de la Universidad de Western Ontario (Canadá) y la Universidad Internacional del Ecuador; así como en Habilidades Docentes en el Instituto Tecnológico de Monterrey (Ecuador). Ha escrito y ha dirigido varios casos reales para el INCAE, la Universidad Internacional del Ecuador, Emprender - Incubadora de Empresas y para sus clases en la universidad.

Obtuvo el título de Máster en Economía Empresarial en el Instituto Centroamericano de Administración de Empresas (Costa Rica) y el de Ingeniero Químico en la Escuela Politécnica Nacional - EPN (Ecuador). 